\title{
Emerging biomarker for predicting acute kidney injury after cardiac surgery: cystatin C
}

\author{
Onur SAYDAM ${ }^{1, \star}$, Ercan TÜRKMEN ${ }^{2}$, Oytun PORTAKAL ${ }^{3}$, Mustafa ARICI $^{2}$, \\ Rıza DOĞAN ${ }^{1}$, Metin DEMİRCİN ${ }^{1}$, İlhan PAŞAOĞLU ${ }^{1}$, Mustafa YILMAZ $^{1}$ \\ ${ }^{1}$ Department of Cardiovascular Surgery, Faculty of Medicine, Hacettepe University, Ankara, Turkey \\ ${ }^{2}$ Department of Nephrology, Faculty of Medicine, Hacettepe University Ankara, Turkey \\ ${ }^{3}$ Department of Biochemistry, Faculty of Medicine, Hacettepe University, Ankara, Turkey
}

Background/aim: Cardiopulmonary bypass (CPB)-associated acute kidney injury (AKI) is a common situation and rapid diagnosis and risk classification are important in the prevention and management of AKI. Changes in serum creatinine (SCr) levels in the current consensus criteria do not allow clinicians to diagnose CPB-associated AKI until $48 \mathrm{~h}$ after surgery.

Materials and methods: We conducted a prospective single center study involving 57 patients who underwent cardiac surgery with CBP to compare serum beta-trace protein (BTP), neutrophil gelatinase-associated lipocalin-2 (NGAL), and cystatin C (CysC) levels with SCr for early diagnosis of CPB associated AKI. We defined AKI according to KDIGO criteria.

Results: AKI was diagnosed in 24 (42.1\%) patients. Mean duration of postoperative intensive care unit stay was 4.79 ( \pm 6.12$)$ days for the $\mathrm{AKI}+$ group and $2.15( \pm 0.56)$ days for the AKI- group. The average length of hospital stay was $8.13( \pm 5.75)$ days for the AKI+ group and 7.21 ( \pm 3.68$)$ days for the AKI- group, which was significantly longer in the AKI+ group $(\mathrm{P}<0.001, \mathrm{P}=0.011)$. Unlike other biomarkers, a significant increase in postoperative CysC levels was always found in patients with AKI during follow-up when compared to patients in whom AKI did not develop $(\mathrm{P}<0.001)$.

Conclusion: Increase in serum CysC levels showed a significant positive correlation with increase in SCr levels. We have not seen this correlation between other biomarkers and SCr. According to our study, serum CysC was a reliable biomarker that may aid in the early detection and follow-up of AKI after cardiac surgery.

Key words: Acute kidney injury, biomarker, cardiac surgical procedures, cystatin C

\section{Introduction}

Acute kidney injury (AKI) is defined as an abrupt decrease in kidney function, resulting in accumulation of urea and other nitrogenous waste products in the body and disruption of the balance of extracellular volume and electrolytes (1). AKI can be seen in $2 \%-30 \%$ of patients having cardiopulmonary bypass $(\mathrm{CPB})$ and $1 \%$ of these patients require renal replacement therapy (RRT) (1). Moreover, CPB-associated AKI is implicated as a major risk factor for prolonged stay in the intensive care unit and hospital and is associated with increased morbidity and mortality. Recently, there have been significant changes in the definition and diagnostic criteria of AKI $(2,3)$. Recent studies showed that acute and milder changes in serum creatinine ( $\mathrm{SCr}$ ) and urine output are associated with significant kidney damage and may lead to serious clinical consequences. SCr is still the most widely used

*Correspondence: onursaydam@hotmail.com

1096 and accepted biomarker for diagnosis of AKI. The most important disadvantage of SCr is its inability to allow an early diagnosis of AKI (4). SCr concentration may not change until approximately $50 \%$ of kidney function has already been lost and it can be affected by sex, muscle mass, diet, and age $(5,6)$. Rapid diagnosis and risk classification are important in management and prevention of disease progression. Identification of reliable biomarkers for early diagnosis of $\mathrm{CPB}$-associated $\mathrm{AKI}$ may increase the chance of successful treatment. Serum beta-trace protein (BTP), neutrophil gelatinase-associated lipocalin-2 (NGAL), and cystatin $\mathrm{C}(\mathrm{Cys} \mathrm{C})$ are currently being investigated for prediction and early diagnosis of AKI, especially in patients undergoing cardiac surgery (7). BTP, which is also known as lipocalin prostaglandin D2 synthase, is a serum biomarker for predicting AKI and was first described as a new biomarker for GFR estimation by Priem et al. (8). 
Because it does not bind to plasma proteins, BTP was filtered almost freely across the glomerular barrier. BTP is synthesized mainly in the central nervous system by glial cells and the choroid plexus and forms one of the principal constituents of the cerebrospinal fluid (9). BTP was shown to have features similar to those of $\mathrm{SCr}$ and $\mathrm{CysC}$ not only in calculation of glomerular filtration rate (GFR) but also in prediction of progressive renal dysfunction (10). NGAL is a protein that is covalently bound to gelatinize in neutrophils. NGAL can be expressed at very low concentrations in several human tissues like kidney, lung, stomach, and colon (11). During AKI, NGAL expression is markedly induced both systemically and locally in injured distal nephron epithelium. Experimental and clinical studies have shown that NGAL could be detected easily in the blood within $3 \mathrm{~h}$ after AKI (12). Therefore, NGAL was shown to be an early, sensitive, and noninvasive biomarker for early detection of AKI in different clinical settings (13). CysC is a cysteine protease inhibitor protein and it is produced in all cells with a nucleus. $\mathrm{Cys} C$ is not bound to plasma proteins and can be freely filtered by the glomerulus. It is reabsorbed and degraded in the proximal tubule and is not secreted into renal tubules (14). Unlike $\mathrm{SCr}, \mathrm{CysC}$ is not affected by age, sex, or mass of muscle cells (15). CysC was investigated in many studies as a predictive biomarker of AKI $(16,17)$. The goal of the present study was to investigate the role of these emerging biomarkers in the early diagnosis and risk prediction for AKI according to KDIGO criteria in adults undergoing cardiac surgery with CPB.

\section{Materials and methods}

In this prospective study, 57 patients among 210 patients who underwent cardiac surgery with $\mathrm{CPB}$ in the Department of Cardiovascular Surgery in Hacettepe University School of Medicine between January 2014 and March 2014 were included. Patients younger than 18 years, patients with chronic kidney disease, and patients who had undergone beating coronary artery bypass surgery were excluded. Surgical re-exploration due to excessive bleeding was also excluded, because CPB was mostly not required in such operations. Patients who had undergone multiple cardiac procedures in a single stage were included. Demographic and preoperative characteristics of the study population were collected including age (years), body surface area $\left(\mathrm{m}^{2}\right)$, additional comorbid problems (diabetes mellitus, hypertension, peripheral arterial disease, autoimmune diseases, obesity, thyroid disease, respiratory disease), preoperative status (elective, urgent, emergent), cardiac diagnosis, total perfusion time, aortic clamp time, and preoperative and postoperative ejection fraction values. Preoperative SCr values were used for evaluation of baseline renal function. In the postoperative period urine output and ejection fraction were measured and occurrence of arrhythmia, delirium, and need for inotropic agent support were noted in all patients. $\mathrm{SCr}$ levels were measured postoperatively at $1,4,12,24$, and 48 h. AKI may occur in the immediate postoperative period as well as throughout the following postoperative days. We defined early AKI as an increase in SCr value of $\geq 0.3$ $\mathrm{mg} / \mathrm{dL}(26.5 \mu \mathrm{mol} / \mathrm{L})$ or $\geq 50 \%$ increase in SCr within $48 \mathrm{~h}$ according to KDIGO criteria (3). Serum samples for BTP, NGAL, and CysC were collected preoperatively (within $48 \mathrm{~h}$ before surgery) and postoperatively at $1,4,12$, and $24 \mathrm{~h}$. Samples were centrifuged at $5000 \times g$ for $10 \mathrm{~min}$ and the supernatants were stored at $-80{ }^{\circ} \mathrm{C}$ until being assessed. Serum BTP concentrations were determined by ELISA (Biovendor, Diagnostics, GmbH, Germany). The measurement range of this method was $0-40 \mathrm{ng} / \mathrm{mL}$ and the method sensitivity was $0.5 \mathrm{ng} / \mathrm{mL}$. Serum NGAL levels were also determined with ELISA (Boster Biological Tech., Fremont, CA, US). Measurement range was 156 to $10,000 \mathrm{pg} / \mathrm{mL}$ and sensitivity of the method was 10 $\mathrm{pg} / \mathrm{mL}$. Cross-reactions have not been reported. Serum $\mathrm{Cys} C$ levels were determined with immunoturbidimetric method (Tina-quant Cystatin C, ROCHE, Diagnostics $\mathrm{GmbH}$, Mannheim, Germany). Measurement range was 0.4 to $8.0 \mathrm{mg} / \mathrm{L}$ with the detection limit (LOD) of 0.40 $\mathrm{mg} / \mathrm{L}$. To study risk factors for AKI in this population, we investigated the relationship between AKI and the risk factors such as mortality, increased postoperative length of stay in intensive care unit (ICU) and hospital, increased duration of mechanical ventilation (MV), and decreased postoperative left ventricular ejection fraction (LVEF). We also recorded the need for RRT. The protocol was approved by the Ethical Committee of Hacettepe University Hospital. All procedures were followed in accordance with the ethical standards of the responsible committee on human experimentation (institutional and national) and with the Helsinki Declaration of 1975, as revised in 2008. Written informed consent was obtained from each patient before being included in the study (18).

\subsection{Statistical analysis}

The data were analyzed using SPSS 21.0. All tests were two-sided, with the alpha level set at 0.05 for statistical significance. Descriptive statistics for categorical variables were reported as frequency and percentage, whereas continuous variables were reported as median [interquartile range (IQR)]. Categorical variables were compared using chi-square tests (with Fisher's exact test when necessary) and continuous variables were compared using the paired Student's t-test, Mann-Whitney U-test, or Kruskal-Wallis test, where appropriate. The normality of the numerical variables was tested by the ShapiroWilks test and the homogeneity of the variances by the Levene test. Nonadjusted logistic regression models 
were used to evaluate the association between AKI and age, postoperative length of stay in ICU, CPB time, and duration of MV. Multivariate logistic regression models were used to predict the outcome variables controlling possible confounding factors.

\section{Results}

In the present study, 20 (35.1\%) female and 37 (64.9\%) male patients were included and the data of a total of 57 patients were analyzed. Mean age of the patients was $56.6 \pm 14.2$. Preoperative values and data for operation procedures for all patients are shown in Table 1. Forty-five patients $(78.9 \%)$ had at least one comorbid disease. The most common comorbid conditions were hypertension (HT) (50.9\%), hyperlipidemia (HL) (49.1\%), obesity (26.3\%), and diabetes mellitus (DM) (19.3\%). The most common surgical procedures in sequence were coronary artery bypass graft surgery (CABG) (59.6\%), mitral valve replacement (MVR) (17.5\%), and aortic valve replacement (AVR) (14.0\%). While 19 patients (33.3\%) needed postoperative inotropic administration, 17 patients (29.8\%) had postoperative atrial fibrillation (AF) and 9 patients (15.8\%) had delirium after the surgery. Three patients $(5.3 \%)$ died because of multiorgan dysfunction. We defined patients having $\mathrm{AKI}$ as $\mathrm{AKI}+$, while patients who did not develop AKI were considered in the AKIgroup. AKI occurred in 24 of the 57 patients (42.1\%). Nonadjusted logistic regression models showed that CPBassociated AKI was associated with increased age (age >
54) $(\mathrm{P}=0.015)$ and comorbidities such as diabetes mellitus, hypertension, and hyperlipidemia $(\mathrm{P}=0.003, \mathrm{P}=0.002$, $\mathrm{P}=0.001$, respectively). AKI was not associated with sex, body mass index, or the type of cardiac procedures, but was associated with increased CPB time. Nonadjusted logistic regression models showed that $\mathrm{CPB}$ time lasting longer than 76 min was significantly associated with development of AKI $(P=0.022)$ (Table 2). The mean duration of postoperative ICU stay was $4.79( \pm 6.12)$ days for the AKI+ group and $2.15( \pm 0.56)$ days for the AKI- group. The average length of hospital stay was $8.13( \pm 5.75)$ days for the AKI+ group and $7.21( \pm 3.68)$ days for the AKI- group, which were significantly longer in AKI+ group $(\mathrm{P}<0.001$, $\mathrm{P}=0.011$ ) (Table 2). As shown in Table 2, preoperative levels of SCr and NGAL were slightly higher in the AKI group but there were no statistically significant differences. On the other hand, preoperative serum levels of CysC ( 1.08 $\pm 0.4 \mathrm{ng} / \mathrm{mL})$ and BTP $(223.1 \pm 45.4)$ were significantly higher in patients with AKI than in patients who did not have AKI $(0.75 \pm 0.19 \mathrm{ng} / \mathrm{mL}$ for CysC and $187.8 \pm 55.1$ for BTP) $(\mathrm{P}<0.001$ and $\mathrm{P}=0.014)$. A significant increase in postoperative $\mathrm{CysC}$ levels was found in patients with AKI in all times when compared to patients in whom AKI did not develop (Figure 1). This increase in serum CysC levels showed a significant positive correlation with increase in SCr levels. We have not seen this correlation between other biomarkers and SCr. CysC had better results for predicting AKI preoperatively when compared to preoperative SCr by using Pearson's correlation test

Table 1. Preoperative values and data for operation procedures for all patients.

\begin{tabular}{|l|l|l|l|l|l|l|}
\hline & $\mathrm{n}$ & $\%$ & Mean & $\begin{array}{l}\text { Standard } \\
\text { deviation }\end{array}$ & Median & Min-max \\
\hline Sex $(\mathrm{F} / \mathrm{M})$ & $20 / 37$ & $35 / 65$ & & & & \\
\hline Age & & & 56.6 & 14.2 & 59 & $18-83$ \\
\hline BSA $\left(\mathrm{m}^{2}\right)$ & & & 1.83 & 0.20 & 1.85 & $1.43-2.21$ \\
\hline CPB flow $(\mathrm{cc})$ & & & 4395.8 & 483.6 & 4440 & $3360-5330$ \\
\hline TPT $(\mathrm{min})$ & & & 88.1 & 43.2 & 83 & $28-170$ \\
\hline ACT $(\mathrm{min})$ & & & 54.1 & 32.0 & 49 & $9-113$ \\
\hline Preop EF $(\%)$ & & 57.9 & 10.1 & 60 & $30-80$ \\
\hline Postop EF (\%) & & & 52.4 & 9.6 & 54 & $20-75$ \\
\hline Preop Beta-trace $(\mathrm{ng} / \mathrm{dL})$ & & & 217.7 & 53.6 & 203.1 & $91.2-308.7$ \\
\hline Preop NGAL $(\mathrm{ng} / \mathrm{dL})$ & & & 43.05 & 12.97 & 46.03 & $7.16-56.13$ \\
\hline Preop cystatin C $(\mathrm{ng} / \mathrm{mL})$ & & & 0.89 & 0.27 & 0.84 & $0.39-1.75$ \\
\hline Creatinine (mg/dL) & & & 0.93 & 0.24 & 0.9 & $0.54-1.82$ \\
\hline BUN & & & 16.3 & 5.5 & 16.6 & $7.5-36.4$ \\
\hline
\end{tabular}

BSA, body surface area; CPB, cardiopulmonary bypass; TPT, total perfusion time; ACT, aortic clamp time; ICU, intensive care unit; EF, ejection fraction; BUN, blood urea nitrogen. 
Table 2. Perioperative data and postoperative length of intensive care unit and hospital stay.

\begin{tabular}{|l|l|l|l|l|l|}
\hline & \multicolumn{2}{l}{} & \multicolumn{2}{l}{ AKI AKI } & \multicolumn{2}{l|}{} \\
\hline & Mean & Std. deviation & Mean & Std. deviation & P \\
\hline CBP flow (cc) & 4363 & 438.9 & 4438 & 542.6 & 0.528 \\
\hline TPT (min) & 76.7 & 28.5 & 102.9 & 53.9 & 0.022 \\
\hline Length of hospital stay (days) & 7.21 & 3.68 & 8.13 & 5.75 & 0.011 \\
\hline Length of ICU stay (days) & 2.15 & 0.56 & 4.79 & 6.12 & $<0.001$ \\
\hline
\end{tabular}

AKI, acute kidney injury; CPB, cardiopulmonary bypass; TPT, total perfusion time; ACT, aortic clamp time

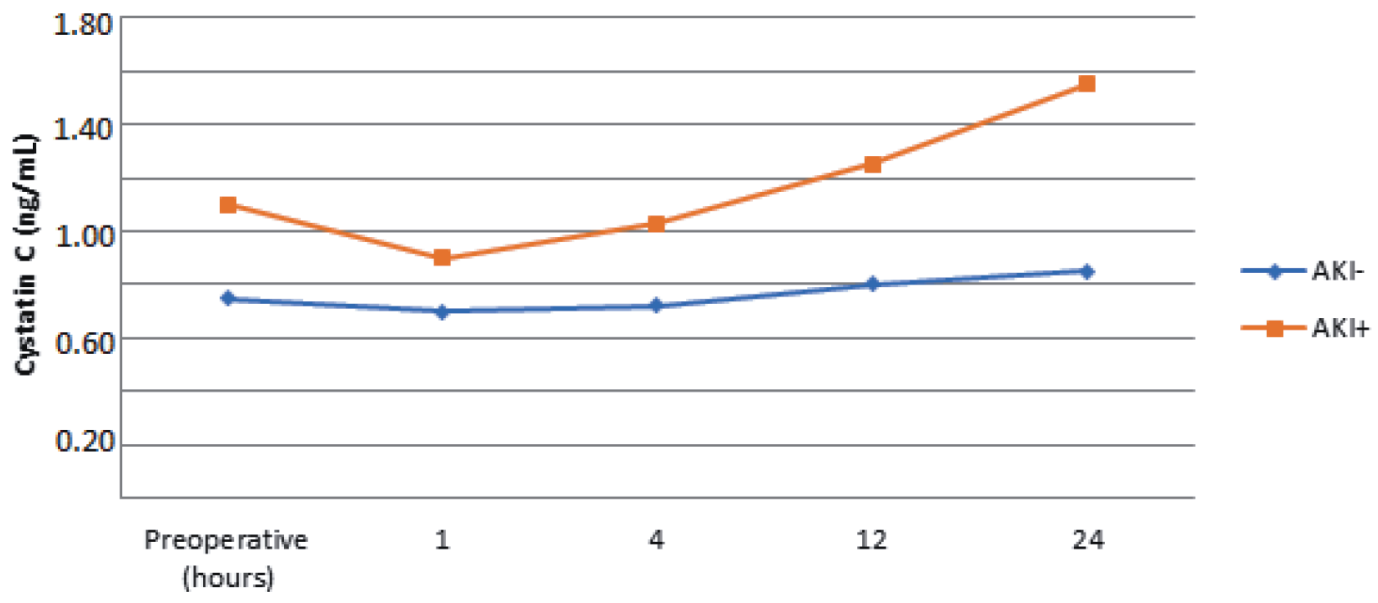

Figure 1. Cystatin C time graph (AKI: acute kidney injury).

(Figure 2). Preoperative levels of serum BTP were $223.1 \pm$ $45.4 \mathrm{ng} / \mathrm{dL}$ in patients with AKI, which was significantly higher than in patients who did not develop AKI $(\mathrm{P}=$ $0.014)$. In subsequent hours after the operation, serum BTP levels were increased in patients with AKI but this was not statistically significant (Figure 3 ). There were no statistically significant differences in preoperative and postoperative serum NGAL levels between the two groups, except for higher levels at $4 \mathrm{~h}$ postoperatively in the AKI+ group (Figure 4). Postoperative development of delirium and atrial fibrillation and the need for administration of inotropic agents were also shown to have an important relation with development of AKI.

\section{Discussion}

The performance of BTP, NGAL, and CysC for early diagnosis of AKI was evaluated separately in previous studies; however, to the best of our knowledge, publications comparing serum levels of these three biomarkers are not available $(16,17,19)$. This is the first study that compares these three biomarkers amongst themselves and with SCr for early diagnosis and monitoring of CPB-associated AKI. This prospective study supports that CysC may allow early detection of CPB-associated AKI before a rise in Scr. Monitoring AKI with CysC may give more reliable results when compared to other new biomarkers and even when compared with the traditional marker SCr. Many independent risk factors for AKI were defined but, in general, causes of AKI are multifactorial. In our study, older patients and patients with hypertension, hyperlipidemia, and diabetes mellitus had higher risk for development of AKI. AKI incidence was $42.1 \%$ in our study. The incidence range of AKI after cardiac surgery was $20 \%-49.9 \%$ according to other studies $(20,21)$. The wide range of incidence of CPB-associated AKI may depend on the definition. Our study was based on KDIGO criteria and they demonstrate greater sensitivity than both AKIN and RIFLE criteria, which may have affected our results. Another reason for the high incidence of AKI may be perfusion modality. We used nonpulsatile flow during $\mathrm{CPB}$ and pulsatile perfusion was found to be superior to nonpulsatile perfusion at preserving microcirculation (22). Moreover, our study showed total perfusion time above 76 min was an important intraoperative risk factor. Sirvinskas et al. (23) also showed in their study that long $\mathrm{CPB}$ duration was a risk factor for development of AKI. 


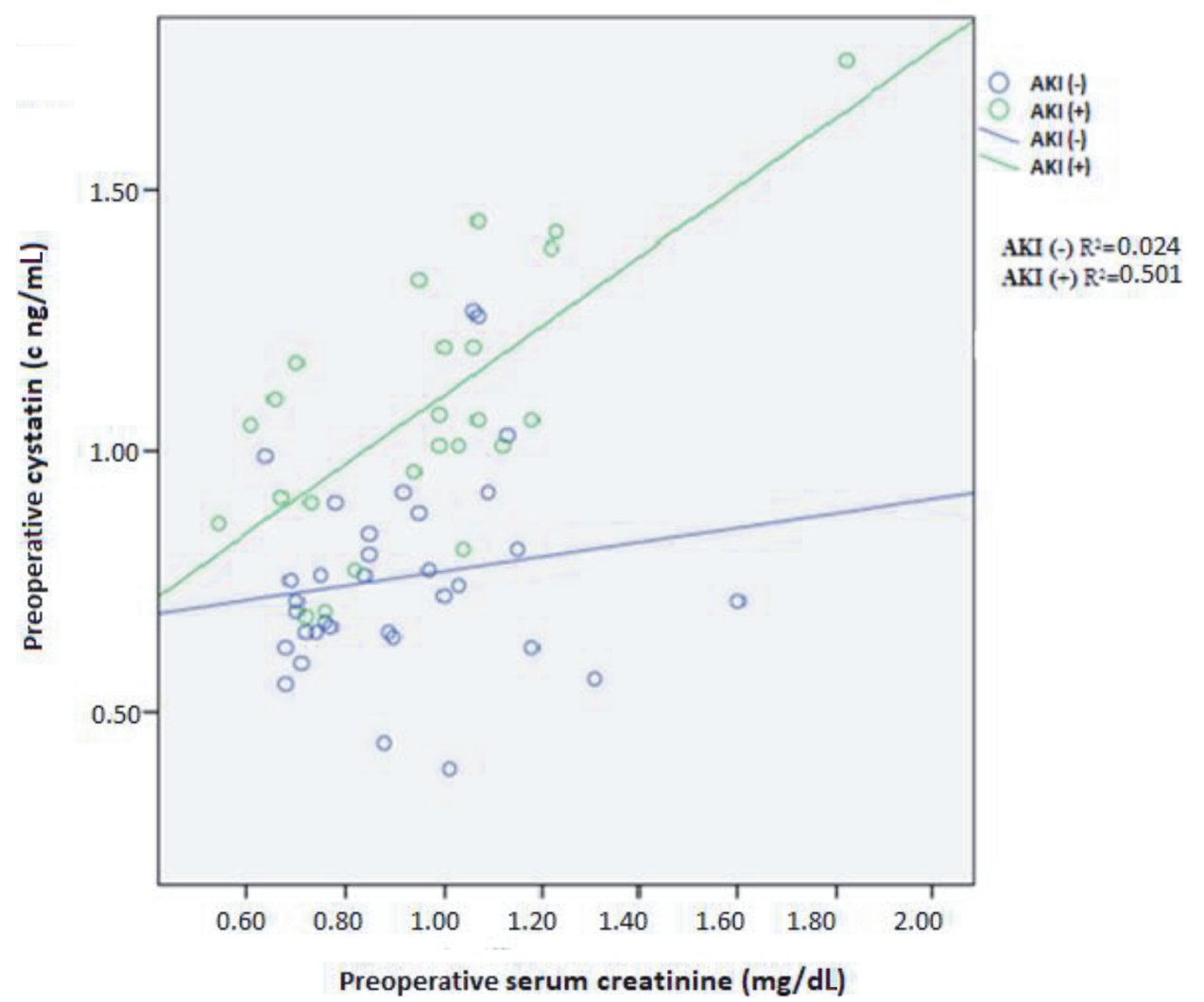

Figure 2. Cystatin $\mathrm{C}$ had better results for predicting AKI preoperatively when compared to preoperative serum creatinine by Pearson's correlation test (AKI: acute kidney injury).

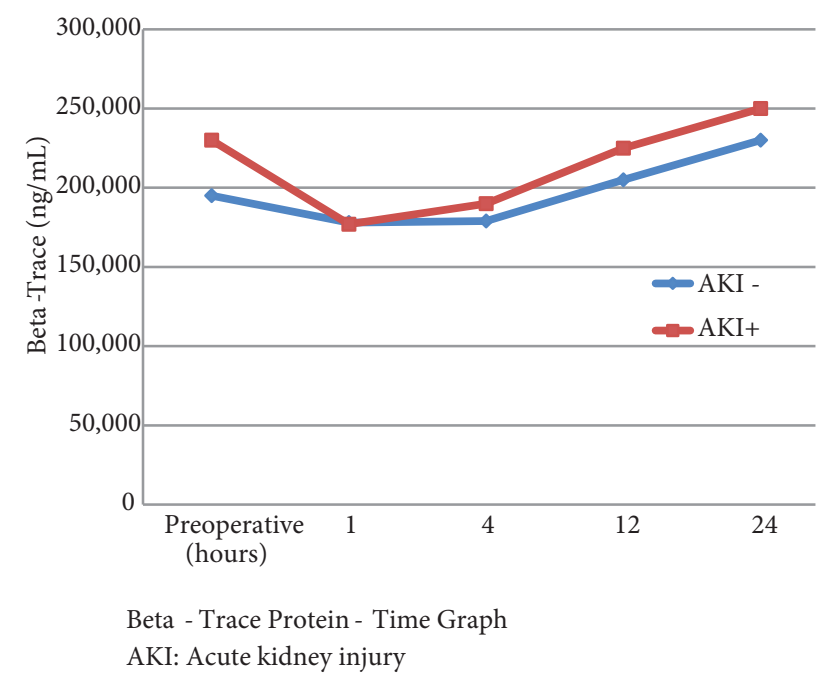

Figure 3. Beta trace protein time graph (AKI: acute kidney injury).

In contrast to our study, Vellinga et al. (24) showed that there was no significant correlation between $\mathrm{CPB}$ duration and AKI development. In our study, CPB-associated AKI was shown to be associated with prolonged stay in the
ICU and increased morbidity and mortality. This may be due to adverse effects of decreased renal function such as electrolyte and volume imbalance, uremia, acidosis, and increased risk of infection (3,25). Postoperative development of delirium and atrial fibrillation and the need for administration of inotropic agents were also shown to have important relations with the development of AKI.

BTP has been studied as a serum or plasma biomarker for assessment of kidney function in patients with kidney disease $(10,19,26)$. Unlike our study, there have been many studies emphasizing good diagnostic performance of BTP as a marker of GFR $(10,27)$. In our study only preoperative values of BTP were significantly higher in patients who developed AKI. Although serum BTP reached higher levels in patients with AKI in subsequent hours after the operation, this was not statistically significant. There are many studies describing similar diagnostic performance of BTP and CysC as a marker of GFR but there is also another study showing better diagnostic performance of CysC than BTP as a GFR marker, which is consistent with our study (28). Although BTP and NGAL showed significant increased levels at different times in the AKI group, those increased levels were not consistent during 


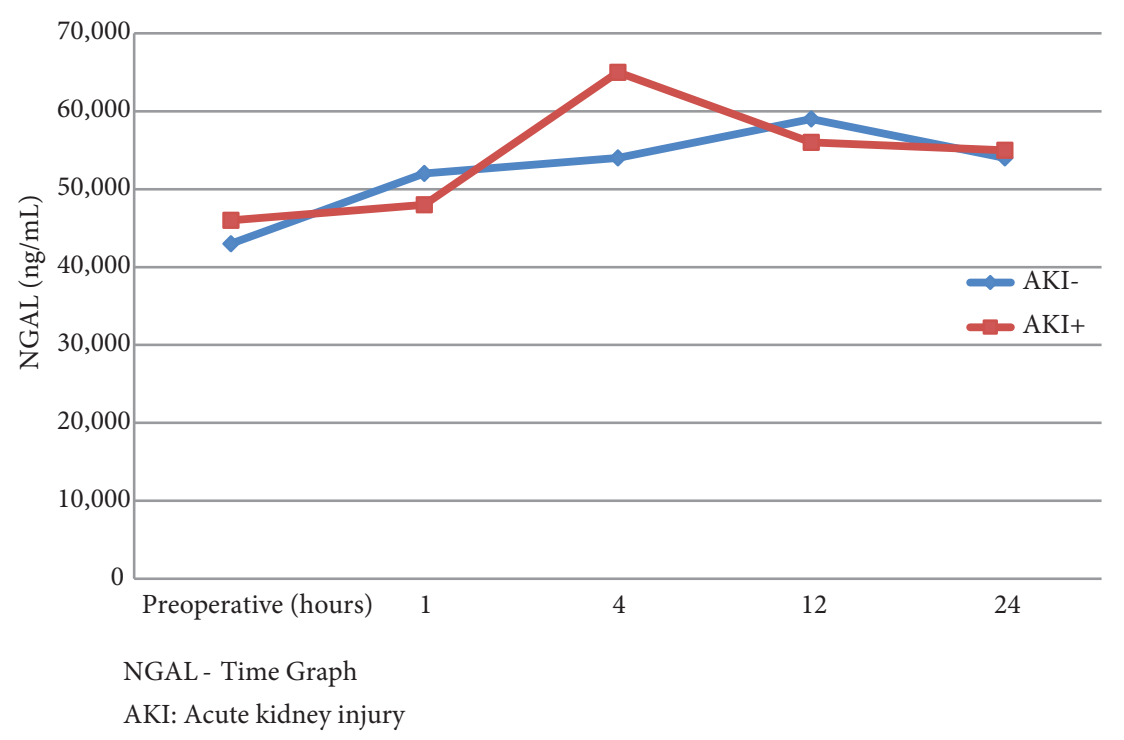

Figure 4. NGAL time graph (AKI: acute kidney injury).

follow up, which prevents us from concluding that these were reliable early diagnostic biomarkers. A large multicenter study reported the early predictive value of NGAL in 1219 adults and found that NGAL levels peaked in urine or plasma within $6 \mathrm{~h}$ after cardiac surgery $(29,30)$. Likewise, according to our study there were no significant differences in preoperative and postoperative levels of serum NGAL between the two groups, except for the levels at $4 \mathrm{~h}$ postoperatively, which had a statistically significant increase. In our study, we found increased serum CysC levels in patients with AKI, which showed a significant positive correlation with the increase in SCr. We have not seen this correlation between other biomarkers and SCr. When preoperative SCr and levels of these new biomarkers are compared, $\mathrm{Cys} C$ had better results for predicting $\mathrm{AKI}$ than the other biomarkers and even SCr. According to Dharnidharka et al. (7), CysC is regarded as an attractive marker in the assessment of GFR. There are also studies reporting lower sensitivity and specificity for $\mathrm{Cys} C$ than for SCr $(31,32)$. Some early precautions can be taken with early diagnosis of renal failure such as temporary cessation of angiotensin converting enzyme inhibitors and angiotensin receptor blockers, optimization of fluid balance, and blood pressure. Although our study achieved its targets, there were some unavoidable limitations. It was conducted in a single institution with a limited sample size. Relatively small sample size might have precluded

\section{References}

1. Rosner MH, Okusa MD. Acute kidney injury associated with cardiac surgery. Clin J Am Soc Nephrol 2006; 1: 19-32. us from detecting minor differences between the groups. Our results have clear clinical and statistical significance but will need to be validated in a larger population. Our study population had relatively normal kidney function and our findings should be confirmed in documented high-risk settings such as urgent surgeries and in patients with preexisting renal dysfunction.

Serum BTP can be used for predicting AKI preoperatively, but postoperative follow-up of AKI with serum BTP is inadequate. Postoperative serum NGAL levels at $4 \mathrm{~h}$ may be used for predicting AKI, but the baseline levels and the levels of the other hours are inadequate. On the other hand, CysC may help for both predicting preoperative risk for development of $\mathrm{AKI}$ and for postoperative follow-up of AKI. As a result, CysC may be used as an early biomarker for the detection of renal dysfunction after cardiac surgery before any detectable increase in SCr level occurs. To overcome the limitations of individual biomarkers, combinations of new and traditional biomarkers should be used together to increase the sensitivity and specificity for predicting AKI.

\section{Acknowledgments}

We would like to thank Professor Saadettin Kılıçkap MD for his assistance with the statistical analyses and Professor Filiz Akbıyık MD for her technical help with the laboratory screening.
2. Mehta RL, Kellum JA, Shah SV, Molitoris BA, Ronco C, Warnock DG, Levin A, Acute Kidney Injury Network. Acute Kidney Injury Network: report of an initiative to improve outcomes in acute kidney injury. Crit Care. 2007; 11: R31. 
3. Acute Kidney Injury Work Group. Kidney Disease: Improving Global Outcomes (KDIGO) Acute Kidney Injury Work Group. KDIGO Clinical Practice Guideline for Acute Kidney Injury. Kidney Inter, Suppl 2012; 2: 1-138.

4. Bellomo R, Kellum JA, Ronco C. Defining acute renal failure: physiological principles. Intensive Care Med 2004; 30: 33-37.

5. Cholongitas E, Shusang V, Marelli L, Nair D, Thomas M, Patch D, Burns A, Sweny P, Burroughs AK. Review article: renal function assessment in cirrhosis - difficulties and alternative measurements. Aliment Pharmacol Ther 2007; 26: 969-978.

6. Gaygisiz U, Aydogdu M, Badoglu M, Boyaci N, Gullu Z, Gursel G. Can admission serum cystatin C level be an early marker subclinical acute kidney injury in critical care patients? Scand J Clin Lab Invest 2016: 1-8.

7. Dharnidharka VR, Kwon C, Stevens G. Serum cystatin C is superior to serum creatinine as a marker of kidney function: a meta-analysis. Am J Kidney Dis 2002; 40: 221-226.

8. Priem F, Althaus H, Birnbaum M, Sinha P, Conradt HS, Jung K. Beta-trace protein in serum: a new marker of glomerular filtration rate in the creatinine-blind range. Clin Chem 1999; 45: 567-568

9. Bökenkamp A, Franke I, Schlieber M, Düker G, Schmitt J, Buderus S, Lentze MJ, Stoffel-Wagner B. Beta-trace proteina marker of kidney function in children: "Original research communication-clinical investigation". Clin Biochem 2007; 40: 969-975.

10. Spanaus KS, Kollerits B, Ritz E, Hersberger M, Kronenberg F, von Eckardstein A, Mild, Moderate Kidney Disease Study Group. Serum creatinine, cystatin C, and beta-trace protein in diagnostic staging and predicting progression of primary nondiabetic chronic kidney disease. Clin Chem 2010; 56: 740-749.

11. Mishra J, Ma Q, Prada A, Mitsnefes M, Zahedi K, Yang J, Barasch J, Devarajan P. Identification of neutrophil gelatinase-associated lipocalin as a novel early urinary biomarker for ischemic renal injury. J Am Soc Nephrol 2003; 14: 2534-2543.

12. Mishra J, Mori K, Ma Q, Kelly C, Barasch J, Devarajan P. Neutrophil gelatinase-associated lipocalin: a novel early urinary biomarker for cisplatin nephrotoxicity. Am J Nephrol 2004; 24: 307-315.

13. Wagener G, Gubitosa G, Wang S, Borregaard N, Kim M, Lee HT. Urinary neutrophil gelatinase-associated lipocalin and acute kidney injury after cardiac surgery. Am J Kidney Dis 2008; 52: 425-433.

14. Kaseda R, Iino N, Hosojima M, Takeda T, Hosaka K, Kobayashi A, Yamamoto K, Suzuki A, Kasai A, Suzuki Y et al. Megalinmediated endocytosis of cystatin $\mathrm{C}$ in proximal tubule cells. Biochem Biophys Res Commun 2007; 357: 1130-1134.

15. Filler G, Bokenkamp A, Hofmann W, Le Bricon T, MartinezBru C, Grubb A. Cystatin C as a marker of GFR-history, indications, and future research. Clin Biochem 2005; 38: 1-8.

16. Nejat M, Pickering JW, Walker RJ, Endre ZH. Rapid detection of acute kidney injury by plasma cystatin $\mathrm{C}$ in the intensive care unit. Nephrol Dial Transplant 2010; 25: 3283-3289.
17. Aydogdu M, Gursel G, Sancak B, Yeni S, Sari G, Tasyurek S, Turk M, Yuksel S, Senes M, Ozis TN. The use of plasma and urine neutrophil gelatinase associated lipocalin (NGAL) and Cystatin $\mathrm{C}$ in early diagnosis of septic acute kidney injury in critically ill patients. Dis Markers 2013; 34: 237-246.

18. Williams JR. The Declaration of Helsinki and public health. Bull World Health Organ 2008; 86: 650-652.

19. Kobata M, Shimizu A, Rinno H, Hamada C, Maeda K, Fukui M, Saito K, Horikoshi S, Tomino Y. Beta-trace protein, a new marker of GFR, may predict the early prognostic stages of patients with type 2 diabetic nephropathy. J Clin Lab Anal 2004; 18: 237-239.

20. Lagny MG, Jouret F, Koch JN, Blaffart F, Donneau AF, Albert A, Roediger L, Krzesinski JM, Defraigne JO. Incidence and outcomes of acute kidney injury after cardiac surgery using either criteria of the RIFLE classification. BMC Nephrol 2015; 16: 76.

21. Ortega-Loubon C, Fernandez-Molina M, Carrascal-Hinojal Y, Fulquet-Carreras E. Cardiac surgery-associated acute kidney injury. Ann Card Anaesth. 2016; 19: 687-698.

22. O’Neil MP, Fleming JC, Badhwar A, Guo LR. Pulsatile versus nonpulsatile flow during cardiopulmonary bypass: microcirculatory and systemic effects. Ann Thorac Surg 2012; 94: 20462053.

23. Sirvinskas E, Andrejaitiene J, Raliene L, Nasvytis L, Karbonskiene A, Pilvinis V, Sakalauskas J. Cardiopulmonary bypass management and acute renal failure: risk factors and prognosis. Perfusion 2008; 23: 323-327.

24. Vellinga S, Verbrugghe W, De Paep R, Verpooten GA, Janssen van Doorn K. Identification of modifiable risk factors for acute kidney injury after cardiac surgery. Neth J Med 2012; 70: 450454.

25. Charlton JR, Portilla D, Okusa MD. A basic science view of acute kidney injury biomarkers. Nephrol Dial Transplant 2014; 29: 1301-1311.

26. Poge U, Gerhardt TM, Stoffel-Wagner B, Palmedo H, Klehr HU, Sauerbruch T, Woitas RP. beta-Trace protein is an alternative marker for glomerular filtration rate in renal transplantation patients. Clin Chem 2005; 51: 1531-1533.

27. Bhavsar NA, Appel LJ, Kusek JW, Contreras G, Bakris G, Coresh J, Astor BC, AASK Study Group. Comparison of measured GFR, serum creatinine, cystatin C, and beta-trace protein to predict ESRD in African Americans with hypertensive CKD. Am J Kidney Dis 2011; 58: 886-893.

28. Solichova P, Novackova L, Ochmanova R, Stejskal D. Assessment of serum beta-trace protein (BTP) measurement in the prediction of glomerular filtration rate. Comparison with serum cystatin C. Biomed Pap Med Fac Univ Palacky Olomouc Czech Repub 2006; 150: 83-84

29. Parikh CR, Devarajan P, Zappitelli M, Sint K, Thiessen-Philbrook H, Li S, Kim RW, Koyner JL, Coca SG, Edelstein CL et al. Postoperative biomarkers predict acute kidney injury and poor outcomes after pediatric cardiac surgery. J Am Soc Nephrol 2011; 22: 1737-1747. 
30. Ghonemy TA, Amro GM. Plasma neutrophil gelatinase-associated lipocalin (NGAL) and plasma cystatin C (CysC) as biomarker of acute kidney injury after cardiac surgery. Saudi J Kidney Dis Transpl 2014; 25: 582-588.

31. Momeni M, Baele P, Jacquet L, Mourad M, Waterloos H, Wallemacq P. Cystatin C in cardiac surgery. Acta Anaesthesiol Belg 2007; 58: 107-112.
32. Spahillari A, Parikh CR, Sint K, Koyner JL, Patel UD, Edelstein CL, Passik CS, Thiessen-Philbrook H, Swaminathan M, Shlipak MG et al. Serum cystatin C- versus creatinine-based definitions of acute kidney injury following cardiac surgery: a prospective cohort study. Am J Kidney Dis 2012; 60: 922-929. 